This item was submitted to Loughborough's Research Repository by the author.

Items in Figshare are protected by copyright, with all rights reserved, unless otherwise indicated.

\title{
Birnbaum's measure of component importance for noncoherent systems
}

PLEASE CITE THE PUBLISHED VERSION

PUBLISHER

(C) IEEE

LICENCE

CC BY-NC-ND 4.0

\section{REPOSITORY RECORD}

Andrews, J.D., and Sally C. Beeson. 2008. "Birnbaum's Measure of Component Importance for Noncoherent Systems”. figshare. https://hdl.handle.net/2134/3807. 
This item was submitted to Loughborough's Institutional Repository (https://dspace.lboro.ac.uk/) by the author and is made available under the following Creative Commons Licence conditions.

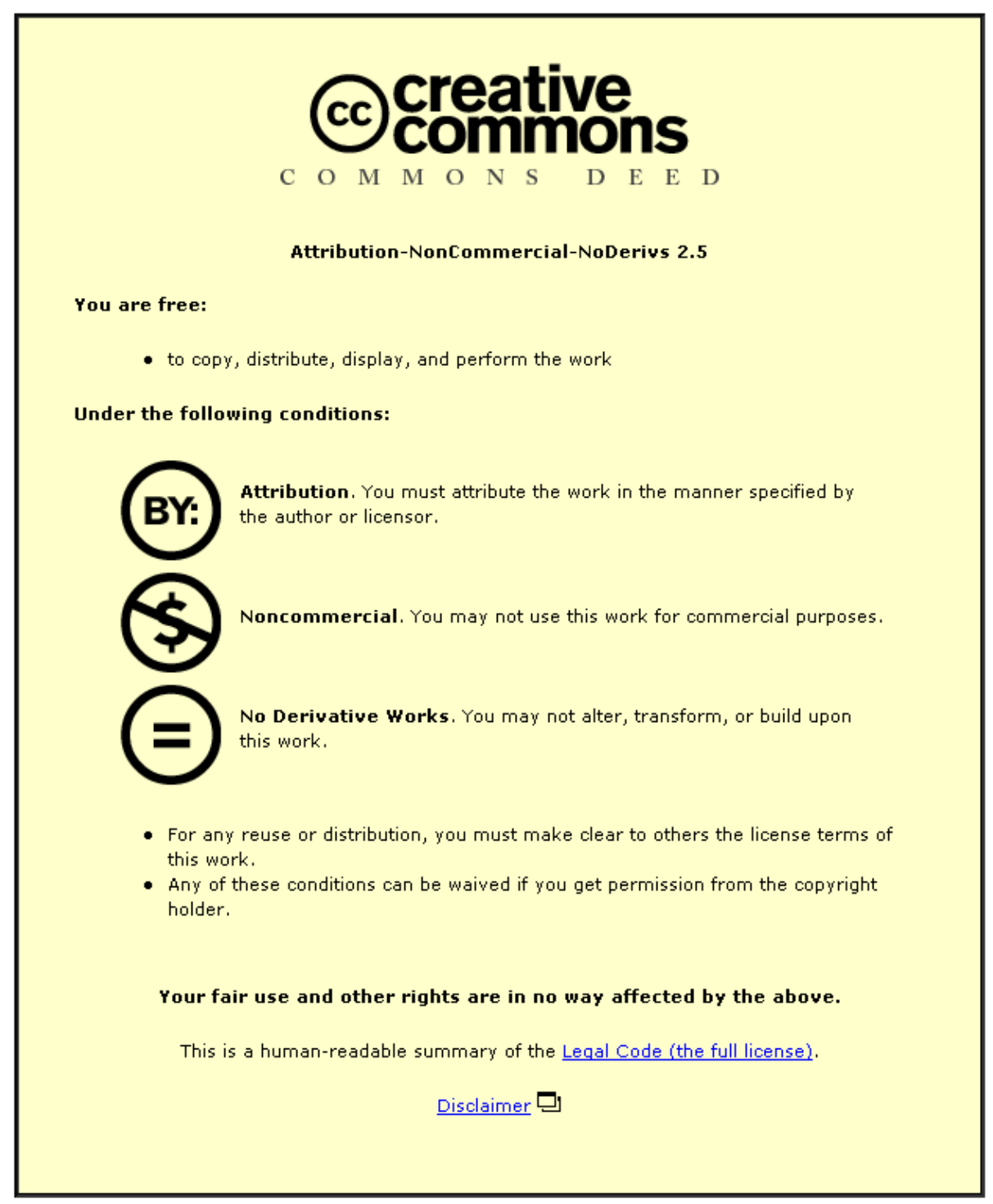

For the full text of this licence, please go to: http://creativecommons.org/licenses/by-nc-nd/2.5/ 


\title{
Birnbaum's Measure of Component Importance for Noncoherent Systems
}

\author{
John D. Andrews and Sally Beeson
}

\begin{abstract}
Importance analysis of noncoherent systems is limited, and is generally inaccurate because all measures of importance that have been developed are strictly for coherent analysis. This paper considers the probabilistic measure of component importance developed by Birnbaum (1969). An extension of this measure is proposed which enables noncoherent importance analysis. As a result of the proposed extension the average number of system failures in a given interval for noncoherent systems can be calculated more efficiently. Furthermore, because Birnbaum's measure of component importance is central to many other measures of importance; its extension should make the derivation of other measures possible.
\end{abstract}

Index Terms-Fault tree, importance measures, noncoherent, structure function.

\section{ACRONYMS}

$\begin{array}{ll}\text { FTA } & \text {-fault-tree analysis } \\ \text { iff } & \text {-if and only if } \\ \text { r.h.s. } & \text { - right-hand side } \\ s- & \quad \text {-statistical(ly) }\end{array}$

\section{NOTATION}

$\phi(x) \quad$ - structure function: defines the system state in terms of the states of the system components

$G_{i}(\underline{q}) \quad$-Birnbaum-measure of component reliability Importance

$G_{i}^{*}(\underline{q}) \quad$-Jackson's proposed-extension of Birnbaum-measure

$G_{i}^{R}(\underline{q}) \quad$-component-repair criticality: $\operatorname{Pr}\{$ the system is in a working state such that repair of component $i$ would cause system failure\}

$G_{i}^{F}(\underline{q}) \quad$-component-failure criticality: $\operatorname{Pr}\{$ the system is in a working state such that failure of component $i$ would cause system failure

$Q_{\mathrm{SYS}}(t) \quad$-system unavailability: $\operatorname{Pr}\{$ the system is in a failed state at time $t\}$

$Q_{\mathrm{SYS}}\left(1_{i}, \underline{q}\right) \quad-\operatorname{Pr}\{$ the system fails with component $i$ failed

$Q_{\mathrm{SYS}}\left(0_{i}, \underline{q}\right) \quad-\operatorname{Pr}\{$ the system fails with component $i$ working\}

Manuscript received February 14, 2001; revised August 2, 2001. Responsible Editor: S. Rai.

The authors are with the Department of Systems Engineering, Loughborough University, Loughborough LE11 3TU UK (e-mail: J.D.Andrews@lboro.ac.uk). Digital Object Identifier 10.1109/TR.2003.809656

${ }^{1}$ The singular and plural of an acronym are always spelled the same.

$q_{i}$
$p_{i}$
$\alpha$
$n$
$n_{p}$
$n_{e}$
$n_{\mathrm{crit}}$
$w_{\mathrm{SYS}}(t)$
$W_{\mathrm{SYS}}$
$w_{i}(t)$
$v_{i}(t)$
$\theta_{i}$
$\epsilon_{i}$
$B$

$B$

\author{
—unavailability of component $i$ \\ - availability of component $i$ \\ —indicator variable with value of 0 or 1 \\ — total number of system components \\ - total number of prime implicants \\ — total number of elements in a selected prime \\ implicant \\ — total number of critical system-states \\ - system unconditional failure intensity \\ $-s$-expected number of system failures in \\ $[0, t)$ \\ - unconditional failure intensity of compo- \\ nent $i$ \\ - unconditional repair intensity of compo- \\ nent $i$ \\ -occurrence of prime-implicant set $\mathbf{i}$ in \\ $[t, t+d t)$ \\ - event that prime implicant $i$ exists at time $t$ \\ - $\operatorname{Pr}\{$ at least 1 prime-implicant set exists at \\ time $t\}$
}

\section{INTRODUCTION}

Definition

OHERENT: A system is coherent if each component is relevant, and the structure function is increasing (nondecreasing).

Safety systems are designed to protect against hazardous events; if failure occurs on a potentially hazardous system, the consequences can be disastrous. Many examples are possible; e.g., the recent crash of the Concord aeroplane in Paris, 2000 July. This left all 113 passengers and crew-members dead. Such disasters make clear the need to minimize the likelihood of system failure. Today, reliability assessment is critical in analyzing and improving system safety.

FTA [1], [2] is a well known and widely used deductive technique developed by Watson in the early 1960s to enable reliability assessment of a wide variety of systems. A fault-tree diagram expresses the causes of a particular system-failure-mode (top event) in terms of component failure modes that are connected by gates (logical operators).

The 3 fundamental gate types used in the fault tree are: AND, OR, and NOT. Generally the use of the NOT gate is discouraged because a fault tree might be noncoherent if the NOT gate is used or is directly implied. In a noncoherent system, component failed states and component working states can contribute to system failure. This can be considered philosophically to be a 
poor analysis because, intuitively, it is a bad design that has components working correctly and contributing to system failure. From a practical viewpoint, the use of NOT logic increases the complexity of analysis and, in some circumstances does not provide additional information about the system. If only the AND and OR gates are used in the fault tree, and all basic events represent failures, then the fault tree is coherent, and only component failures can contribute to system failure.

A fault tree is noncoherent if its structure function $\phi(\underline{x})$ does not comply with the definition of coherence given by the properties of relevance and monotonicity [3], [4]:

1) Every component $i$ is relevant: $\phi\left(1_{i}, \underline{x}\right) \neq \phi\left(0_{i}, \underline{x}\right)$ for some $\underline{x}$.

2) The structure function of component $i$ is monotonically increasing: $\phi\left(1_{i}, \underline{x}\right) \geq \phi\left(0_{i}, \underline{x}\right), \forall i, \forall \underline{x}$. $\phi(1, \underline{x}) \equiv \phi\left(x_{1}, \ldots, x_{i-1}, 1, x_{i+1}, \ldots, x_{n}\right)$; $\phi(0, \underline{x}) \equiv \phi\left(x_{1}, \ldots, x_{i-1}, 0, x_{i+1}, \ldots, x_{n}\right)$.

- Condition \#1 ensures that each component contributes to the system state.

- Condition \#2 an increasing (nondecreasing) structure function, ensures that the system state deteriorates (at least does not improve) with an increasing number of component failures.

- Component failures cannot improve the system state.

Although the use of NOT logic is often discouraged, [5] demonstrates that for multi-tasking systems, NOT logic is essential if successful and meaningful analysis is to be performed. This is also true for event-tree analysis [6]-[8]. Hence it is essential to consider NOT logic for such systems and be able to analyze resulting noncoherent fault trees efficiently and accurately.

FTA can be split into 2 stages:

1) Qualitative analysis-identifies the minimal cut sets or, for noncoherent fault trees, the prime-implicant sets (minimal combinations of both success and failure events that cause the top event).

2) Quantitative analysis-involves calculating the system unavailability and the system unreliability; and can involve analysis of component and minimal cut set (prime implicant) importance.

Importance analysis, and Birnbaum's measure of component reliability importance in particular, is the focus of this paper.

\section{BIRNBAUM'S COMPONENT IMPORTANCE}

When assessing a system, its performance depends on that of its components. Some components play a more important role in causing or contributing to system failure than others. The concept of importance measures is to numerically-rank the contribution of each component, or basic event, to reflect the susceptibility of the system to the occurrence of this event. Importance-measures assign a numerical value between 0 and 1 to each system component; 1 signifies the highest level of importance.

Reference [9] introduced the concept of importance, and developed a probabilistic measure of component-reliability importance. $G_{i}(q)$ is defined as: the probability that component $i$ is critical to system failure; i.e., when $i$ fails it causes the system to pass from a working-state to a failed-state. Birnbaum's measure is also referred to as the criticality function:

$$
G_{i}(\underline{q})=Q_{\mathrm{SYS}}\left(1_{i}, \underline{q}\right)-Q_{\mathrm{SYS}}\left(0_{i}, \underline{q}\right)
$$

$Q_{\mathrm{SYS}}\left(1_{i}, \underline{q}\right) \equiv \operatorname{Pr}\{$ system fails with component $i$ failed $\}$, $Q_{\mathrm{SYS}}\left(0_{i}, \underline{q}\right) \equiv \operatorname{Pr}\{$ system fails with component $i$ working $\}$, $\underline{q} \equiv$ vector of component unavailability's for the remaining components.

$Q_{\mathrm{SYS}}$ is generated using the inclusion-exclusion expansion (and is considered in greater detail later).

Although this field has received much attention over the past 30 years, the majority of measures that have been developed, have been developed specifically for the analysis of coherent systems, and therefore have ranked component failures. Importance analysis of noncoherent systems is extremely limited; it is generally inaccurate and misleading because importance is approximated using the measures developed for the analysis of coherent systems.

Reference [10] considers the extension of some of the most commonly used measures of importance to enable analysis of noncoherent systems; it begins by developing an extension of Birnbaum's measure and then uses this extension to extend other measures based on Birnbaum's measure. This proposed extension of Birnbaum's measure is:

$$
G_{i}^{*}(\underline{q})=\left|Q_{\mathrm{SYS}}\left(1_{i}, \underline{q}\right)-Q_{\mathrm{SYS}}\left(0_{i}, \underline{q}\right)\right| .
$$

It is not clear exactly how this measure should be interpreted; (2) suggests only 1 calculation per component. However, in a worked example, [10] actually ranks component failure importance and component repair importance separately. To demonstrate the difficulties encountered with this extension, consider the basic noncoherent system that [10] uses during this worked example. The system has 3 prime-implicant sets: $\{a, b\},\{a, c\} .\{b, \bar{c}\}$. While the system details are not important for this demonstration, they are in [11].

The calculation procedure in [11] can be used to obtain an expression for the system unavailability function of a noncoherent system. The calculation procedure is

$$
\begin{array}{r}
Q_{\mathrm{SYS}}(t)=\sum_{i=1}^{n_{p}} \operatorname{Pr}\left\{\epsilon_{i}\right\}-\sum_{i=1}^{n_{p}-1} \sum_{j=i+1}^{n_{p}} \operatorname{Pr}\left\{\epsilon_{i} \cap \epsilon_{j}\right\}+\cdots \\
+(-1)^{n_{p}-1} \cdot \operatorname{Pr}\left\{\epsilon_{1} \cap \epsilon_{1} \cap \cdots \epsilon_{n_{p}}\right\}
\end{array}
$$

$\alpha=1$ if event $i$ is a member of $\epsilon_{i}, \alpha=0$ if event $\bar{i}$ is a member of $\epsilon_{i}$.

$$
\begin{aligned}
\operatorname{Pr}\left\{\epsilon_{i}\right\} & =\prod_{j=1}^{n_{e}} q^{\alpha_{j}}(t), \\
q^{\alpha_{j}}(t) & = \begin{cases}q_{j}, & \text { if } \alpha=1 \\
p_{j}, & \text { if } \alpha=0\end{cases}
\end{aligned}
$$

$\alpha=1$ if a normal literal occurs, $\alpha=0$ if a negated literal occurs.

Rather than perform Boolean reduction with terms involving both failed and success states, the following identity is used: $p_{i} \cdot q_{i}=0$. 
TABLE I

The RESUlTS OBTAINED IN [10]

\begin{tabular}{c|cc}
\hline Event & Results & Ranking \\
\hline$a$ & $5.665 \cdot 10^{-3}$ & 4 \\
$b$ & $8.105 \cdot 10^{-3}$ & 3 \\
$c$ & $9.575 \cdot 10^{-3}$ & 2 \\
$\bar{c}$ & $3.839 \cdot 10^{-2}$ & 1 \\
\hline
\end{tabular}

Hence, the following expressions are obtained from (3):

$$
\begin{aligned}
\sum_{i=1}^{n_{p}} \operatorname{Pr}\left\{\epsilon_{i}\right\} & =\sum_{i=1}^{3} \operatorname{Pr}\left\{\epsilon_{i}\right\} \\
& =q_{a} \cdot q_{b}+q_{a} \cdot q_{c}+q_{b} \cdot p_{c}, \\
\sum_{i=1}^{n_{p}-1} \sum_{j=i+1}^{n_{p}} \operatorname{Pr}\left\{\epsilon_{i} \cap \epsilon_{j}\right\} & =\sum_{i=1}^{2} \sum_{j=2}^{3} \operatorname{Pr}\left\{\epsilon_{i} \cap \epsilon_{j}\right\} \\
& =q_{a} \cdot q_{b} \cdot q_{c}+q_{a} \cdot q_{b} \cdot p_{c}, \\
\operatorname{Pr}\left\{\epsilon_{1} \cap \epsilon_{2} \cap \epsilon_{3}\right\} & =0 \cdot 0 .
\end{aligned}
$$

Thus:

$$
Q_{\mathrm{SYS}}(t)=q_{a} \cdot q_{b}+q_{a} \cdot q_{c}+q_{b} \cdot p_{c}-q_{a} \cdot q_{b} \cdot q_{c}-q_{a} \cdot q_{b} \cdot p_{c} .
$$

The component unavailability's assigned in [10] are $p_{i} \equiv 1-q_{i}$, $q_{a}=9.90099 \cdot 10^{-3}, q_{b}=3.84615 \cdot 10^{-2}, q_{c}=1.52534 \cdot$ $10^{-2}$. The results, using these numbers, in [10] for $G_{i}^{*}(\underline{q})$ are in Table I.

An alternative way of considering Birnbaum's measure for this example is to consider component-criticality by an exhaustive tabular approach. Consider a system with $n$ components; the system state can then be expressed in terms of the component states. It is possible to determine whether a component is critical to system failure, given the states of the remaining $n-1$ components. There are possible $2^{n-1}$ states of the other $n-1$ components. By identifying the critical situations for component $i$ and summing their probabilities of occurrence, one can calculate the probability that component $i$ is critical to system failure.

Thus for the example in [10], Table II identifies the critical states for each of the 3 components. Table III records the sum of the critical situations for each event, the probability that each event is critical to system failure, and the ranking that each component receives.

Comparing the results from [10] in Table I with those in Table III it is clear that not only does the extension [10] calculate component criticality incorrectly but that it also ranks the components incorrectly. Hence it the extension [10] is not conceptually equivalent.

\section{EXTENSION OF BIRNBAUM'S MEASURE OF COMPONENT IMPORTANCE FOR NONCOHERENT ANALYSIS}

Birnbaum's measure of component reliability importance (importance is defined as: probability that component $i$ is critical to system failure) is the fundamental probabilistic measure of importance. Many other measures of importance are extensions of this measure. Birnbaum developed this measure only for the analysis of coherent systems. It is calculated from the system unavailability function, $Q_{\mathrm{SYS}}(t)$, which is obtained using the exclusion-inclusion principle and Boolean reduction laws. $G_{i}(\underline{q})$ can be evaluated from (1) which, because $Q_{\mathrm{SYS}}(t)$ is linear in each $q_{i}$, can be expressed as:

$$
G_{i}(\underline{q})=\frac{\partial Q_{\mathrm{SYS}}(t)}{\partial q_{i}(t)} .
$$

Because, for coherent systems, Birnbaum's measure is central to so many other measures of importance, its extension to enable analysis of noncoherent systems must provide a consistent foundation to extend these measures for noncoherent analysis.

When dealing with a coherent system, system failure can only be caused by component failure. Hence a component in a coherent system can only be failure-critical. However, when dealing with a noncoherent system, system failure can be caused, not only by component failure (referred to as event $i$ ), but also by component repair (referred to as event $\bar{i}$. Thus a component in a noncoherent system can be failure-critical or repair-critical. These two criticalities must be considered separately because component $i$ can exist in only 1 state at any time.

The probability required is: the probability that component $i$ is critical to system failure; this can be expressed as $G_{i}^{R}(\underline{q})$ or $G_{i}^{F}(\underline{q})$.

$$
G_{i}(\underline{q})=G_{i}^{R}(\underline{q})+G_{i}^{F}(\underline{q}) .
$$

An expression for the system unavailability function can be obtained from calculation procedure outlined in (3) [11]. Component $i$ is failure critical if the system is working, but the system fails if component $i$ fails. Thus the probability that component $i$ is failure critical is the probability that the system is in a working state such that the failure of component $i$ causes at least 1 prime-implicant set containing event $i$ to occur. This probability is calculated by obtaining the probability that at least 1 prime-implicant set containing event $i$ exists at time $t$ and then dividing this probability by the unavailability of component $i$.

To calculate this probability it is helpful to re-express the system unavailability in the 3 distinct terms:

$$
Q_{\mathrm{SYS}}(t)=i \cdot A+\bar{i} \cdot B+C \text {. }
$$

These 3 terms represent, respectively,

- those products involving the failure of component $i$,

- those products involving the repair of component $i$,

- those products for which component $i$ is irrelevant.

The probability that component $i$ is failure critical is:

$$
G_{i}^{F}(\underline{q})=\frac{\operatorname{Pr}\{i \cdot A\}}{q_{i}}=\operatorname{Pr}\{A\} .
$$

Similarly, the probability that component $i$ is repair critical is the probability that the system is in a working state such that the repair of component $i$ causes at least 1 prime-implicant set containing event $\bar{i}$ to occur. This is calculated by obtaining the probability that at least 1 prime-implicant set containing event 
TABLE II

POSSIBLE AND CRITICAL STATES FOR THE EVENTS

\begin{tabular}{ccc|ccc|ccc}
\hline $\begin{array}{c}\text { State of } \\
a\end{array}$ & $\begin{array}{c}\text { State of } \\
b\end{array}$ & Is $c$ & State of & State of & Is $b$ & State of & State of & Is $a$ \\
Critical & $a$ & $c$ & Critical & $b$ & $c$ & Critical \\
\hline W & W & No & W & W & Yes (F) & W & W & No \\
W & F & Yes (R) & W & F & No & W & F & Yes (F) \\
F & W & Yes (F) & F & W & Yes (F) & F & W & No \\
F & F & No & F & F & No & F & F & Yes (F) \\
\hline
\end{tabular}

$\mathrm{W}=$ Working,$\quad \mathrm{F}=$ Failed

TABLE III

EXPECTED RESULTS

\begin{tabular}{c|ccc}
\hline Event & Sum of Critical Situations & Expected Result & Rank \\
\hline$a$ & $p_{b} \cdot q_{c}+q_{b} \cdot q_{c}$ & 0.152534 & 2 \\
$b$ & $p_{a} \cdot p_{c}+q_{a} \cdot p_{c}$ & 0.84747 & 1 \\
$c$ & $q_{a} \cdot p_{b}$ & 0.00952 & 4 \\
$\bar{c}$ & $p_{a} \cdot q_{b}$ & 0.03808 & 3 \\
\hline
\end{tabular}

$\bar{i}$ exists at time $t$ and then dividing this probability by the availability of component $i$.

$$
G_{i}^{R}(\underline{q})=\frac{\operatorname{Pr}\{\bar{i} \cdot B\}}{p_{i}}=\operatorname{Pr}\{B\} .
$$

The top event can only exist at time $t$ if at least 1 prime-implicant set exists at time $t$. Hence, the repair and failure criticalities can be calculated separately by differentiating $Q_{\mathrm{SYS}}(t)$ with respect to $p_{i}$ and $q_{i}$, respectively.

$$
\begin{aligned}
& G_{i}^{F}(\underline{q})=\frac{\partial Q_{\mathrm{SYS}}(t)}{\partial q_{i}}, \\
& G_{i}^{R}(\underline{q})=\frac{\partial Q_{\mathrm{SYS}}(t)}{\partial p_{i}},
\end{aligned}
$$

(if $Q_{\mathrm{SYS}}(t)$ is expressed according to the method in [11]).

\section{Example}

Consider the noncoherent system introduced in Section III, the Boolean expression for the top event is:

$$
T=a \cdot b+a \cdot c+b \cdot \bar{c} .
$$

The system unavailability is in (4). The proposed extension can be used to calculate the repair and failure importance of any component. The failure importance and repair importance for component $c$ are calculated from (9) and (10):

$$
\begin{aligned}
& G_{c}^{F}(\underline{q})=q_{a}-q_{a} \cdot q_{b}=q_{a} \cdot p_{b} \\
& G_{c}^{R}(\underline{q})=q_{b}-q_{a} \cdot q_{b}=q_{b} \cdot p_{a} .
\end{aligned}
$$

Hence, from (6):

$$
G_{c}(\underline{q})=p_{a} \cdot q_{b}+q_{a} \cdot p_{b} .
$$

This result can be checked by using the tabular approach introduced in Section III. There are 4 situations for which component $c$ could be FAILURE or REPAIR critical to the system failure according to the states of components $a$ and $b$. Table IV outlines the 4 situations; column \#3 records whether component $c$ is critical to system failure.
TABLE IV

CRITICALITY ASSESSMENT FOR COMPONENT $c$

\begin{tabular}{ccc}
\hline $\begin{array}{c}\text { State of } \\
a\end{array}$ & $\begin{array}{c}\text { State of } \\
\text { W }\end{array}$ & Is $c$ \\
critical \\
\hline W & W & No \\
W & F & Yes (REPAIR) \\
F & W & Yes (FAILURE) \\
F & F & No \\
\hline
\end{tabular}

From Table IV it is clear that component $c$ is critical for 2 of the 4 situations, hence Birnbaum's measure for component $c$ is calculated as:

$$
G_{c}(\underline{q})=\sum_{k=1}^{n_{\text {crit }}} \operatorname{Pr}\{\text { Critical Situation } k\}=p_{a} \cdot q_{b}+q_{a} \cdot p_{b} .
$$

The result obtained using this tabular approach is the same as the result obtained using the proposed equation. The proposed extension calculates the probability that component $i$ is critical to system failure. Having calculated the component repair and failure criticality, components need to be ranked, and the results analyzed; this is considered in Section V.

\section{EXPECTED NUMBER OF SYSTEM FAILURES}

The expression for calculating the $s$-expected number of system failures, $W_{\mathrm{SYS}}(0, t)$, in $[0, t]$, when the analysis is coherent can be given in terms of Birnbaum's measure of component reliability importance [12].

$$
W_{\mathrm{SYS}}(0, t)=\int_{0}^{t} \sum_{i=1}^{n} G_{i}(\underline{q}) \cdot w_{i}(u) d u \text {. }
$$

This identity can be extended to noncoherent systems as follows:

$$
\begin{aligned}
& W_{\mathrm{SYS}}(0, t) \\
& =\int_{0}^{t}\left[\sum_{i=1}^{n} G_{i}^{F}(\underline{q}) \cdot w_{i}(u)+\sum_{i=1}^{n} G_{i}^{R}(\underline{q}) \cdot v_{i}(u)\right] d u .
\end{aligned}
$$

On the r.h.s. of (14), term \#1 calculates the $s$-expected number of occurrences of system failure due to the failure of component $i$ in a given interval, and term \#2 calculates the $s$-expected number of occurrences of system failure in the given interval due to the repair of component $i$.

If this extension of Birnbaum's importance measure has the desired properties, then (14) holds. This section tests this extension by considering a basic example and comparing the results 
obtained for $W_{\mathrm{SYS}}(0, t)$ using a method developed in [11] and using (14).

The procedure in [11] works directly with the Boolean expression for the top event obtained from qualitative analysis.

$$
W_{\mathrm{SYS}}(0, t)=\int_{0}^{t} w_{\mathrm{SYS}}(u) d u \text {. }
$$

"System unconditional failure intensity" $\equiv \operatorname{Pr}\{$ top event occurs at $t$ /unit-time $\}$. The top event occurs in $[t, t+d t)$ iff none of the prime-implicants sets exist at $t$, and at least 1 prime-implicant set occurs in $[t, t+d t)$. The unconditional failure intensity is:

$$
w_{\mathrm{SYS}}(t) d t=\operatorname{Pr}\left\{\bigcup_{i=1}^{n} \theta_{i}\right\}-\operatorname{Pr}\left\{B \cdot \bigcup_{i=1}^{n} \theta_{i}\right\} .
$$

Note: There is an important distinction between existence and occurrence. If a prime implicant exists at time $t$, then all component states in the prime implicant must have occurred prior to $t$. Whereas, if a prime implicant occurs at time $t, 1$ component-state must occur in $[t, t+d t)$.

The first term of (16) represents the probability that at least 1 prime-implicant set occurs in $[t, t+d t)$. The second term of (16) is a correction term that calculates the probability that at least 1 prime-implicant set occurs in $[t, t+d t)$ but do not fail the system because it is already failed because at least 1 implicant set already exists.

The unconditional failure intensity is calculated for the example in Section II with the Boolean expression for the top event.

$$
T=a \cdot b+a \cdot c+b \cdot \bar{c} .
$$

The 2 terms of (16) are calculated separately. Term \#1 on the r.h.s. of (16) can be expressed using the inclusion-exclusion expansion to give:

$$
\begin{aligned}
\operatorname{Pr} & \left\{\bigcup_{i=1}^{3} \theta_{i}\right\} \\
= & \sum_{i=1}^{3} \operatorname{Pr}\left\{\theta_{i}\right\}-\sum_{i=1}^{2} \sum_{j=i+1}^{3} \operatorname{Pr}\left\{\theta_{i} \cdot \theta_{j}\right\}+\operatorname{Pr}\left\{\theta_{1} \cdot \theta_{2} \cdot \theta_{3}\right\} \\
= & \operatorname{Pr}\left\{\theta_{1}\right\}+\operatorname{Pr}\left\{\theta_{2}\right\}+\operatorname{Pr}\left\{\theta_{3}\right\}-\operatorname{Pr}\left\{\theta_{1} \cdot \theta_{2}\right\} \\
& \quad-\operatorname{Pr}\left\{\theta_{1} \cdot \theta_{3}\right\}-\operatorname{Pr}\left\{\theta_{2} \cdot \theta_{3}\right\}+\operatorname{Pr}\left\{\theta_{1} \cdot \theta_{2} \cdot \theta_{3}\right\} \\
= & {\left[w_{a} \cdot q_{b}+w_{b} \cdot q_{a}+w_{a} \cdot q_{c}+w_{c} \cdot q_{a}+w_{b} \cdot p_{c}+v_{c} \cdot q_{b}\right.} \\
& \left.\quad-\left(q_{b} \cdot q_{c} \cdot w_{a}+q_{a} \cdot p_{c} \cdot w_{b}\right)\right] d t \\
= & {\left[w_{a} \cdot\left(q_{b}+q_{c}-q_{b} \cdot q_{c}\right)+w_{b} \cdot\left(q_{a}+p_{c}-q_{a} \cdot p_{c}\right)\right.} \\
& \left.+w_{c} \cdot q_{a}+v_{c} \cdot q_{b}\right] d t .
\end{aligned}
$$

Similarly, expanding term \#2 on the r.h.s. of (16) gives:

$$
\begin{aligned}
\operatorname{Pr}\left\{B \cdot \bigcup_{i=1}^{3} \theta_{i}\right\} & =\sum_{i=1}^{3} \operatorname{Pr}\left\{\theta_{i}, B\right\} \\
& -\sum_{i=1}^{2} \sum_{j=i+1}^{3} \operatorname{Pr}\left\{\theta_{i} \cdot \theta_{j} \cdot B\right\}+\operatorname{Pr}\left\{\theta_{1} \cdot \theta_{2} \cdot \theta_{3} \cdot B\right\} .
\end{aligned}
$$

Each term is also expanded about $B$ thus, for example, expansion of term \#1 gives:

$$
\begin{aligned}
\operatorname{Pr}\left\{\theta_{1} \cdot B\right\}= & \operatorname{Pr}\left\{\theta_{1} \cdot \epsilon_{1}\right\}+\operatorname{Pr}\left\{\theta_{1} \cdot \epsilon_{2}\right\}+\operatorname{Pr}\left\{\theta_{1} \cdot \epsilon_{3}\right\} \\
& -\operatorname{Pr}\left\{\theta_{1} \cdot \epsilon_{1} \cdot \epsilon_{2}\right\}-\operatorname{Pr}\left\{\theta_{1} \cdot \epsilon_{1} \cdot \epsilon_{3}\right\} \\
& -\operatorname{Pr}\left\{\theta_{1} \cdot \epsilon_{2} \cdot \epsilon_{3}\right\}+\operatorname{Pr}\left\{\theta_{1} \cdot \epsilon_{1} \cdot \epsilon_{2} \cdot \epsilon_{3}\right\} .
\end{aligned}
$$

Treating other terms in the same way gives:

$$
\begin{aligned}
\operatorname{Pr}\left\{\theta_{1} \cdot B\right\} & =w_{b} \cdot q_{a} \cdot q_{c}+w_{a} \cdot q_{b} \cdot p_{c} ; \\
\operatorname{Pr}\left\{\theta_{2} \cdot B\right\} & =w_{c} \cdot q_{a} \cdot q_{b} ; \\
\operatorname{Pr}\left\{\theta_{3} \cdot B\right\} & =v_{c} \cdot q_{a} \cdot q_{b} ; \\
\sum_{i=1}^{2} \sum_{j=i+1}^{3} \operatorname{Pr}\left\{\theta_{i} \cdot \theta_{j} \cdot B\right\} & =0 \\
\operatorname{Pr}\left\{\theta_{1} \cdot \theta_{2} \cdot \theta_{3} \cdot B\right\} & =0 .
\end{aligned}
$$

Hence

$$
\begin{aligned}
W_{\mathrm{SYS}}(0, t)= & \int_{0}^{t} w_{\mathrm{SYS}}(u) d u \\
w_{\mathrm{SYS}}(u)= & w_{a}\left(q_{b}+q_{c}-q_{b} \cdot\left(q_{c}+p_{c}\right)\right) \\
& +w_{b}\left(q_{a}+p_{c}-q_{a} \cdot\left(q_{c}+p_{c}\right)\right) \\
& +w_{c}\left(q_{a}-q_{a} \cdot q_{b}\right)+v_{c}\left(q_{b}-q_{a} \cdot q_{b}\right) \\
W_{\mathrm{SYS}}(0, t)= & \int_{0}^{t}\left[w_{a} \cdot q_{c}+w_{b} \cdot p_{c}\right. \\
& \left.\quad+w_{c} \cdot q_{a} \cdot p_{b}+v_{c} \cdot q_{b} \cdot p_{a}\right] d u .
\end{aligned}
$$

Use the extended expression in (14) to calculate the $s$-expected number of system failures. The system unavailability for this example is given in (4). Hence:

$$
\begin{aligned}
& G_{a}^{F}(\underline{q})=q_{b}+q_{c}-q_{b} \cdot\left(q_{c}+p_{c}\right)=q_{c}, \\
& G_{a}^{R}(\underline{q})=0, \\
& G_{b}^{F}(\underline{q})=q_{a}+p_{c}-q_{a} \cdot\left(q_{c}+p_{c}\right)=p_{c}, \\
& G_{b}^{R}(\underline{q})=0 \\
& G_{c}^{F}(\underline{q})=q_{a} \cdot\left(1-q_{b}\right)=q_{a} \cdot p_{b}, \\
& G_{c}^{R}(\underline{q})=q_{b} \cdot\left(1-q_{a}\right)=q_{b} \cdot p_{a} .
\end{aligned}
$$

From (14),

$$
\begin{aligned}
W(0, t) & =\int_{0}^{t} w_{\mathrm{SYS}}(u) d u \\
& =\int_{0}^{t}\left[w_{a} \cdot q_{c}+w_{b} \cdot p_{c}+w_{c} \cdot q_{a} \cdot p_{b}+v_{c} \cdot q_{b} \cdot p_{a}\right] d u .
\end{aligned}
$$

The $s$-expected number of system failures is the same for both of the calculation procedures, demonstrating that,

1) the identity in (13) can be extended, as shown in (14), for noncoherent analysis;

2) the proposed measure calculates the desired probability. 


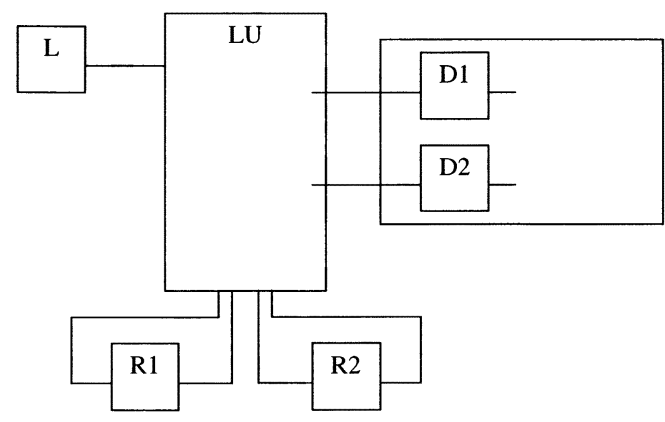

Fig. 1. Simplified gas detection system.

\section{Gas Detection System Example}

Consider the simplified gas-detection system in Fig. 1. This is a multi-tasking system introduced in [5]. The system has 2 sensors, D1 and D2, which detect leakage in a confined space. The detectors send signals along individual cables to the computer logic control unit, LU. If the LU receives a signal that there is a gas leak from any sensor, then 3 functions must be performed:

- Process shut down: de-energize relay R1,

- Inform the operator of the leak, using a lamp and siren labeled L,

- Remove the power supply from affected areas: de-energize relay $\mathrm{R} 2$.

Reference [5] considers one particular failure scenario wherein, although the operator is informed of the gas release, both the "process shut-down" and the "power-supply isolation" fail. It was shown that NOT logic was essential if successful analysis was to be performed. The fault tree for this particular mode of failure has 2 prime-implicant sets, $\{\overline{\mathrm{L}}, \overline{\mathrm{LU}}, \mathrm{R} 1, \mathrm{R} 2, \overline{\mathrm{D} 1}\},\{\overline{\mathrm{L}}, \overline{\mathrm{LU}}, \mathrm{R} 1, \mathrm{R} 2, \overline{\mathrm{D}} 2\}$.

To illustrate the method used to analyze component importance, availability values have been assigned to each component: $q_{\mathrm{L}}=0.01, q_{\mathrm{LU}}=0.04, q_{\mathrm{R} 1}=q_{\mathrm{R} 2}=0.06, q_{\mathrm{D} 1}=q_{\mathrm{D} 2}=0.02$.

The system unavailability is:

$Q_{\mathrm{SYS}}(t)=p_{\mathrm{L}} \cdot p_{\mathrm{LU}} \cdot q_{\mathrm{R} 1} \cdot q_{\mathrm{R} 2} \cdot q_{\mathrm{D} 1}+p_{\mathrm{L}} \cdot p_{\mathrm{LU}} \cdot q_{\mathrm{R} 1} \cdot q_{\mathrm{R} 2} \cdot q_{\mathrm{D} 2}$

$$
-p_{\mathrm{L}} \cdot p_{\mathrm{LU}} \cdot q_{\mathrm{R} 1} \cdot q_{\mathrm{R} 2} \cdot q_{\mathrm{D} 1} \cdot q_{\mathrm{D} 2} \text {. }
$$

From (9) and (10)

$$
\begin{aligned}
G_{\mathrm{L}}^{F}(\underline{q}) & =0 \\
G_{\mathrm{L}}^{R}(\underline{q}) & =p_{\mathrm{LU}} \cdot q_{\mathrm{R} 1} \cdot q_{\mathrm{R} 2} \cdot\left(p_{\mathrm{D} 1}+p_{\mathrm{D} 2}-p_{\mathrm{D} 1} \cdot p_{\mathrm{D} 2}\right) \\
& =0.00346, \\
G_{\mathrm{LU}}^{F}(\underline{q}) & =0, \\
G_{\mathrm{LU}}^{R}(\underline{q}) & =p_{\mathrm{L}} \cdot q_{\mathrm{R} 1} \cdot q_{\mathrm{R} 2} \cdot\left(p_{\mathrm{D} 1}+p_{\mathrm{D} 2}-p_{\mathrm{D} 1} \cdot p_{\mathrm{D} 2}\right) \\
& =0.00358, \\
G_{\mathrm{R} 1}^{F}(\underline{q}) & =G_{\mathrm{R} 2}^{F}(\underline{q}) \\
& =p_{\mathrm{L}} \cdot p_{\mathrm{LU}} \cdot q_{\mathrm{R} 2} \cdot\left(p_{\mathrm{D} 1}+p_{\mathrm{D} 2}-p_{\mathrm{D} 1} \cdot p_{\mathrm{D} 2}\right) \\
& =0.057, \\
G_{\mathrm{R} 1}^{R}(\underline{q}) & =G_{\mathrm{R} 2}^{R}(\underline{q})=0,
\end{aligned}
$$

TABLE $\mathrm{V}$

RESULTS AND RANKING FOR TOTAL CRITICALITY

\begin{tabular}{c|cc}
\hline Event & $\begin{array}{c}\text { Total } \\
\text { Criticality }\end{array}$ & Ranking \\
\hline L & 0.00346 & 3 \\
LU & 0.00358 & 2 \\
R1 & 0.057 & 1 \\
R2 & 0.057 & 1 \\
D2 & 0.00007 & 4 \\
D1 & 0.00007 & 4 \\
\hline
\end{tabular}

TABLE VI

RESULTS AND RANKING FOR FAILURE CRITICALITY

\begin{tabular}{c|cc}
\hline & Failure & \\
Event & Criticality & Ranking \\
\hline L & 0 & N/A \\
LU & 0 & N/A \\
R1 & 0.057 & 1 \\
R2 & 0.057 & 1 \\
D1 & 0 & N/A \\
D2 & 0 & N/A \\
\hline
\end{tabular}

TABLE VII

RESULTS AND RANKING FOR REPAIR CRITICALITY

\begin{tabular}{c|cc}
\hline & Repair & \\
Event & Criticality & Ranking \\
\hline L & 0.00346 & 2 \\
LU & 0.00358 & 1 \\
R1 & 0 & N/A \\
R2 & 0 & N/A \\
D1 & 0.00007 & 3 \\
D2 & 0.00007 & 3 \\
\hline
\end{tabular}

$$
\begin{aligned}
G_{\mathrm{D} 1}^{F}(\underline{q}) & =G_{\mathrm{D} 2}^{F}(\underline{q})=0, \\
G_{\mathrm{D} 1}^{R}(\underline{q}) & =G_{\mathrm{D} 2}^{R}(\underline{q})=p_{\mathrm{L}} \cdot p_{\mathrm{LU}} \cdot q_{\mathrm{R} 1} \cdot q_{\mathrm{R} 2} \cdot\left(1-p_{\mathrm{D} 2}\right) \\
& =0.00007 .
\end{aligned}
$$

Tables V-VII record the results and ranking for the total criticality, the failure criticality, and the repair criticality.

Table $\mathrm{V}$ records the total criticality of each component and the ranking obtained. From this table, the system is most likely to be in a critical state for components R2 and R1. The importance of components LU and L are closely numerically ranked 2 nd and 3rd respectively. The failure and repair criticality of each component are given in Tables VI and VII, respectively.

Components R1 and R2 are ranked highest and can only be failure critical. From this ranking, the system is most likely to be in a working but critical state for components R1 and R2. Should system performance be inadequate, then 2 steps can be taken to increase system reliability.

1. The likelihood of this critical state occurring for either R1 or R2 can be reduced. In general this can be achieved by increasing the availability of any components whose failure is necessary for component $i$ to be failure critical.

2. The availability of components R1 and R2 can be increased to reduce the likelihood of either causing system failure.

Components LU and $\mathrm{L}$ were ranked 2 nd and 3rd highest, but both components can only be repair-critical. Thus if the system 
is in a state such that components LU and L are repair critical, it is vital that they are not repaired to a working state until the system-state changes and they are not repair critical. It is not appropriate to reduce the availability of components that can be repair critical, instead.

The probability of existence of the necessary and sufficient conditions for the component to be repair-critical needs to be minimized.

The repair of a component which can be repair-critical needs to be done at an appropriate time, i.e., when it is not repaircritical (other component failures repaired first).

\section{REFERENCES}

[1] E. J. Henley and H. Kumamoto, Probabilistic Risk Management: IEEE Press, 1991.

[2] J. D. Andrews and T. R. Moss, Reliability and Risk Assessment, 2nd ed: ASME, 2002.

[3] R. E. Barlow and F. Proschan, Mathematical Theory of Reliability: Wiley, 1965

[4] A. Bendell and J. Ansell, "The incoherency of multistate coherent systems," Reliab. Eng., vol. 8, pp. 165-178, 1984.

[5] J. D. Andrews, "The use of Not logic in fault tree analysis," Qual. Reliab. Eng., vol. 17, pp. 143-150, 2001.

[6] I. A. Papazoglou, "Mathematical foundations of event trees," Qual. Reliab. Eng. Int., vol. 17, pp. 143-150, 2001.

[7] J. D. Andrews and S. Dunnett, "Improving accuracy in event tree analysis," in Foresight and Precaution, Proc. ESREL 2000, SARS and SRA-EUROPE Annual Conf., Cottam, Harvey, Pape, and Tait, Eds., May 2000.

[8] J. D. Andrews and S. J. Dunnett, "Event tree analysis using binary decision diagrams," IEEE Trans. Rel., vol. R-49, no. 2, pp. 230-239, Jun. 2000 .
[9] Z. W. Birnbaum, "On the importance of different components in a multicomponent system," in Multivariate Analysis, P. R. Krishnaiah, Ed: Academic Press, 1969, vol. 11.

[10] P. S. Jackson, "On the $s$-importance of elements and prime implicants of noncoherent systems," IEEE Trans. Rel., vol. R-32, no. 1, pp. 21-25, Apr. 1983.

[11] T. Inagaki and E. J. Henley, "Probabilistic evaluation of prime implicants and top events for noncoherent systems," IEEE Trans. Rel., vol. R-29, no. 5 , pp. 361-367, Dec. 1980.

[12] T. Aven and U. Jensen, Stochastic Models in Reliability: Springer, 1999, p. 113.

John D. Andrews is a Professor in the Department of Systems Engineering at Loughborough University. He recently joined this department from the Department of Mathematical Sciences. He joined the Loughborough University in 1989, having gained 9 years industrial research experience with British Gas, and 2 years lecturing experience in the Mechanical Engineering Department at the University of Central England. His current research interests concern the assessment of the safety and risk of potentially hazardous industrial activities. This research has been heavily supported by industrial funding. In recent years, grants have been secured from the MOD, Rolls Royce Aero Engines, Mobil North Sea, and Bechtel. Professor Andrews has numerous journal/conference publications along with a jointly authored book Risk and Reliability Assessment which is now in its second edition.

Sally Beeson graduated from Loughborough University in 1999 with a first class honors degree. She has continued her studies at Loughborough by undertaking research in Risk and Reliability quantification methods. Her research project has focused on the analysis of noncoherent systems using Binary Decision Diagrams. Sally has been involved in developing new importance measures for noncoherent systems, and how these can be used in the efficient calculation of the system-failure frequency. 